\title{
Visual Outcome After Antioxidant Supplementation
}

Dear Editor:

In 2001, the National Eye Institute-funded Age-related Eye Disease Study (AREDS) published its findings. ${ }^{1}$ The AREDS was a double-masked, randomized, placebo-controlled trial of 4757 subjects over a period of 5 years that showed that supplementation with antioxidants vitamins $\mathrm{C}$ and $\mathrm{E}, \beta$-carotene, and zinc in combination resulted in a $25 \%$ relative reduction in the risk of progression from intermediate to advanced and visually consequential age-related macular degeneration (AMD). The AREDS supplement did not contain the macular pigment carotenoids lutein and zeaxanthin, compounds believed to confer protection against development and/or progression of AMD because of their antioxidant and/or optical (blue light-filtering) properties. There is an emerging body of opinion that deficiency of macular pigment might play an etiological role in the development of AMD. ${ }^{2}$ We report the results of the Carotenoids in Age-related Maculopathy study (ISRCTN number 94557601), a randomized, double-blind, placebo-controlled clinical trial of lutein and zeaxanthin with co-antioxidants versus placebo in persons at high risk of progression to late AMD. ${ }^{3}$

Participants were aged $\geq 55$ years with features of early AMD in both eyes (group 1) or exhibiting late stage features of choroidal neovascularization or geographic atrophy in only 1 eye (group 2). Randomization was double masked and participants were assigned to receive an oral antioxidant preparation that consisted of a tablet taken twice a day to deliver a daily dose of $12 \mathrm{mg}$ of lutein, $0.6 \mathrm{mg}$ zeaxanthin, $15 \mathrm{mg} d$ - $\alpha$-tocopherol (vitamin E), $150 \mathrm{mg}$ ascorbic acid (vitamin C), $20 \mathrm{mg}$ zinc oxide, and $0.4 \mathrm{mg}$ copper gluconate, or placebo. The levels of these components were lower than that used in AREDS owing to regulatory restrictions in the European Union. Of the 433 participants who entered the study, 216 participants were assigned to receive the active preparation and 217 placebo. Two hundred fifty-two of the participants belonged to group 1 contributing 2 study eyes to the analysis. The remaining 181 contributed a sole study eye.

We originally chose the difference in photopic interferometric acuity between groups as the primary outcome measure at 12 months. However, this parameter was found to be unreliable and at the recommendation of the independent data and safety monitoring committee we changed it to best-corrected visual acuity (BCVA).

Participants underwent a full, general physical examination, and collection of demographic information at baseline. These included measurement of height, weight, sitting blood pressure, and completion of a set of questionnaires that included information on diet and lifestyle. Ocular examinations were undertaken at all study visits, which occurred at baseline and at 6 monthly intervals, and included BCVA, photopic interferometry, contrast sensitivity, shape discrimination testing, fundus photography, and Raman spectroscopy.

There were no imbalances in any of the measured baseline variables by treatment assignment. Eighty-eight participants (20\%) exited the study before the 12-month visit and were equally distributed between the 2 arms. The mean change in BCVA was -0.1 (standard deviation [SD] 7.0) in the treatment group and -0.3 (SD 7.7) in the placebo group. This differential change was not significant.

Although no difference in BCVA (which was the primary outcome measure) was found between the supplemented and placebo groups at 12 months, a number of secondary outcomes suggested functional benefits in the interventional arm and are consistent with emerging reports from other studies. ${ }^{4}$ These are the subject of a further detailed report. ${ }^{5}$

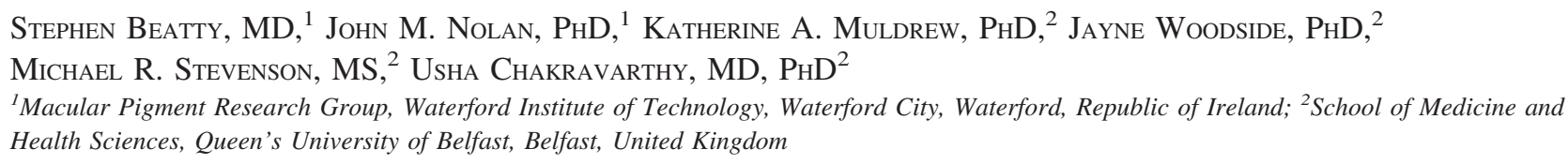

References

1. The AREDS research group. A randomized, placebo-controlled, clinical trial of high-dose supplementation with vitamins $C$ and $E$, beta carotene, and zinc for age-related macular degeneration and vision loss: AREDS report no. 8. Arch Ophthalmol 2001;119:1417-36.

2. Loane E, Kelliher C, Beatty S, Nolan JM. The rationale and evidence base for a protective role of macular pigment in age-related maculopathy. Br J Ophthalmol 2008;92:1163-8.

3. Neelam K, Hogg RE, Stevenson MR, et al. Carotenoids and co-antioxidants in age-related maculopathy: design and methods. Ophthalmic Epidemiol 2008;15:389-401.

4. Weigert G, Kaya S, Pemp B, et al. Effects of lutein supplementation on macular pigment optical density and visual acuity in patients with age-related macular degeneration. IOVS 2011;52:8174-8.

5. Beatty S, Nolan JM, Muldrew KA, et al. Secondary outcomes in a clinical trial of carotenoids with co-antioxidants versus placebo in early age-related macular degeneration. Ophthalmology 2013;120:600-6. 